\title{
Children's Early Educational Game Under the Background of Chinese Three Kingdoms Culture - To Borrow Arrows with Thatched Boats
}

\author{
Hui Liang \\ Department of Software \\ Engineering \\ Zhengzhou University of Light \\ Industry \\ Zhengzhou, China \\ hliang@zzuli.edu.cn
}

\author{
Fanyu Bao \\ Department of Software \\ Engineering \\ Zhengzhou University of Light \\ Industry \\ Zhengzhou, China \\ 953413891@qq.com \\ Mingge Pan \\ Department of Arts and Design \\ Zhengzhou University of Light \\ Industry \\ Zhengzhou, China \\ pmg-1@163.com
}

\author{
Yusheng Sun \\ Department of Software \\ Engineering \\ Zhengzhou University of Light \\ Industry \\ Zhengzhou, China \\ 1987004@zzuli.edu.cn \\ Jian Chang \\ National Centre for Computer \\ Animation \\ Bournemouth University \\ Poole, UK \\ jchangsuper@163.com
}

\author{
Qian Zhang \\ Department of Arts and Design \\ Zhengzhou University of Light \\ Industry \\ Zhengzhou, China \\ qianzhang@zzuli.edu.cn
}

\begin{abstract}
Recent research has shown that children's strategic ability can be improved with game. To improve strategic ability, motor coordination ability and the understanding of historical knowledge, we design an ability training game - to borrow arrows with thatched boats. In order to improve children's learning efficiency, we use deep motion perception technology as a human-computer interaction tool. Our system can allow children to control the movement of boats and interact with virtual objects in the virtual environment through hand-gesture. We can provide some strategic options, such as weather, equipment, etc. Under this new mode of educational game, children's strategic and motor coordination abilities are improved, and their understanding of historical knowledge can be also enhanced.
\end{abstract}

Keywords—Chinese culture, educational game, virtual reality

\section{INTRODUCTION}

Traditional Chinese culture, the driving force of social development, incorporates the essence of all eras over thousands of years. The popularization of traditional culture education for all children in the society is extremely important in today's time. Currently, most children are learning traditional culture only at the level of memorization, but fail to achieve deep understanding. Due to the diversity of learning methods, students are often misled by the erroneous history presented in historical novels, plays, costume dramas, etc. At the same time, these models that do not involve students in the learning process are uninteresting to them, and it is difficult for students to gain historical knowledge, cognitive skills, and coordination skills from these models. It is possible to improve our knowledge of history and coordination ability at any age. However, many studies have shown that cognitive abilities do not change or improve much in adulthood and that the early years of development are a short critical period [1]-[5]. Therefore, we chose children aged 6-12 years old as the test subjects for the ability enhancement training. Although a great deal of research has been conducted on competency enhancement training, there is still a lot of room for development in this area. Few people use virtual reality devices to provide real-time interaction in order to adjust the impact of teaching and learning. In this context, we need new forms and means of teaching.

Acquiring knowledge should be an enjoyable process for children, not a monotonous exercise. Children are unknowingly drawn to new things from birth. How to make them learn effortlessly during their childhood is a debatable question. Just as aromatic food arouses people's appetite, exquisite merchandise will inflame customers' desire for purchase, and games will foment their lust for entertainment. Educational gamification is a commonly used teaching strategy today. The value of educational games is demonstrated by the fact that children's abilities improve as they complete the game [6].

Games should provide students with fresh experiences and aid them to improve various skills [7]. Achievement goals are an important indicator of the game, the degree of achievement we achieve through tasks and challenges [8], which regards as a driving force. Different people reach different achievement goals depending on their circumstances. As users complete each challenge, they acquire new knowledge and abilities. The psychological state generated by the player after the game will vary with the outcome [9]. Many a time, players are willing to continue due to the courage and confidence that a higher score brings. Conversely, facing a low score produces two very different psychological states: demoralization or inspired determination. 
The special definition of serious games brings innovative expectations to traditional Chinese cultural education. The way to immerse children in virtual reality games has excellent interactive effect in educational games. This paper selects the classic battle of the Three Kingdoms period (Figure 1 [10]), Borrowing Arrows with Straw Boats, designs and implements an educational game named To Borrow Arrows with Thatched Boats which based on hand-gesture interaction with this background.

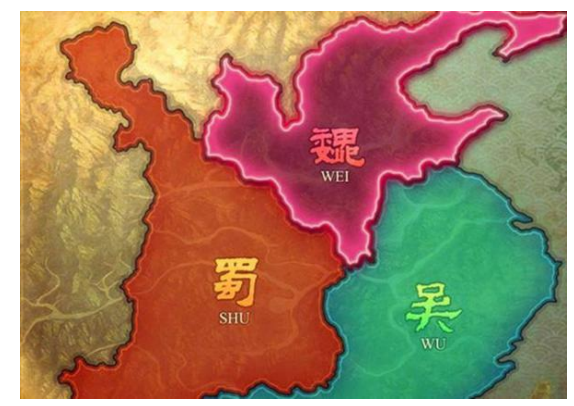

Fig. 1. Three Kingdoms.

Borrowing Arrows with Straw Boats is a famous story of the Battle of the Red Cliff in The Romance of the Three Kingdoms. Zhuge Liang (an outstanding politician and military strategist of the ShuHan group) was to be executed if he failed to fulfill his promise to Zhou $\mathrm{Yu}$ (an outstanding military strategist of the DongWu group) to produce 100,000 arrows in ten days. One foggy night, Zhuge Liang, taking advantage of Cao Cao's suspicious character, let Cao's army shoot arrows at the straw boats, successfully completed the mission. Using this historical story as an educational game background will not only broaden their knowledge of history, but also enhance their thinking abilities and coordination abilities.

This paper proposes a novel model of early childhood education that combines traditional Chinese culture with modern technology. Since the game-based education and the novel natural gesture interaction method are willingly accepted by children, they can be better trained in several abilities: enhancing children's historical knowledge, strengthening children's strategic skills, and training hand, eye and brain coordination skills. This paper will be discussed in the following six sections: The first part is Introduction, which introduces the research background of this paper and why we designed To Borrow Arrows with Thatched Boats. The second part is Related Work, which mainly introduces the fields of using virtual reality and what kind of role VR plays in this article. The third part is Implementation, which describes the design perspective of To Borrow Arrows with Thatched Boats, including the techniques used and the goals hoped to be achieved. The fourth part is Results, which describe the effect of the game through a three-dimensional framework. The fifth part is Experiment and Discussion. We invited 70 children to test this serious game, and the results show that it achieved the effect we expected. The sixth part is Conclusion, which is a summary of the whole paper.

\section{RELATED WORK}

Virtual reality technology has been involved in many fields such as entertainment [11], military [12], medicine [13], film and television [14], and especially has a wide range of applications in the education industry. Virtual reality as a carrier for the expression of pedagogical knowledge offers more possibilities for the medium of teaching and learning. With immersive learning environments, more intuitive imagery and more advanced interactive representations, these technologies engage the senses of teachers and students in the teaching process.

In traditional geography teaching, books, films and videos are frequently used as the medium for teaching and explaining to students. In addition to field trips, it is difficult to provide students with a more thorough understanding and appreciation of what they are learning through other teaching methods. The use of virtual reality design activities in the assessment of science courses can fill this gap. The results of the study showed that students who performed virtual reality design activities had better learning outcomes and improved selfefficacy and critical thinking skills [15]. In the study by Tüzün $\mathrm{H}$ [16], students were allowed to learn about continents and countries of the world through qualitative and quantitative methods. The results show that students have high intrinsic motivation to learn in the virtual reality game environment. With less emphasis on grades, students are more committed to learning geographic knowledge and understanding.

With the help of virtual reality, we understand art in an immersive way, thus overcoming the abstraction and obscurity of art. Children experiment with their own hands to learn "how people painted over the centuries, how Renaissance workshops were created, and how crayon and watercolor painting techniques of the eighteenth century worked [17]." 3D museums are also used as one of the learning tools to acquire more knowledge about art history [18]. We also have software that teaches singing like Sing Master [19]. Applying VR technology in art education can make teaching more intuitive, which is conducive to inspiring students and enhancing their enthusiasm for learning.

History will not reemerge until virtual reality technology emerges. Photo-realistic Byzantine church models generated using VR technology can be applied for scientific analysis, preservation-restoration and conservation purpose [20]. Etruscanning $3 \mathrm{D}$ is a gesture-based interactive project that tells the user the history of archaeological finds and their importance through storytelling [21]. History is characterized by past, richness, and comprehensiveness, and its content is not verifiable or recoverable, but historical scenes can be recreated again. The introduction of virtual reality technology in history teaching has achieved the purpose of making cultural relics speak and history "come alive". Students explore on their own with the teacher's guidance, touching the pulse of history and stimulating curiosity, wonder and imagination.

Since traditional Chinese culture is vast and profound, this requires us to make greater efforts in inheriting and preserving it. The new teaching method of integrating advanced science and technology into the two-dimensional teaching mode can allow traditional culture to break through the limitations of time and space [22]. This article focuses on traditional Chinese culture to design a serious game designed to teach children about Chinese history in a relaxed environment. A teaching 
method that combines traditional elements with modern technology can foster children's interest in Chinese culture. Achieving maximum user immersion and providing the most satisfying educational experience [23] is the ultimate goal of our game design.

\section{SYSTEM DESIGN}

Knowledge acquisition/content understanding as well as emotional and motivational outcomes are common impacts from educational games [24]. Head-mounted virtual reality devices enable players to isolate reality from the virtual so they can focus on the game situation and have the same experience as solving real-world problems. The sense of substitution in the game will enhance the interest of the players. Virtual reality can solve the problem of people not being able to experience historical scenes due to the irreversibility of history. Players can participate in historical stories by reconstructing historical scenes across time and space to fully stimulate their bodily perception system and initiative, allowing participants to better understand history [25].

Borrowing Arrows with Straw Boats is a form of presenting historical stories based on the game. It uses the interactive mode provided by advanced technology to allow children to enter the Three Kingdoms and simulate Zhuge Liang's decision-making scheme. This mode of entertainment through immersive virtual reality [26] not only allows children to learn history, but also develops students' strategic and coordination skills. Players' subjective experiences in games are central to explaining game engagement: process [27], [28], immersion [29], existence [30], and awakening [31].

Our game design focuses on three main areas, one is the understanding of history, the second is the development of children's strategic ability, and motor coordination ability. Before the game starts, we can understand the history more deeply by introducing the synopsis of the story and the actual operation in the game. The game tasks include gestural manipulation of the game objects according to the game requirements and selection of the objects suitable for driving the game plot. For these three aspects of training, we give out different test indicators.

To address the design priorities, we will work on the following areas:

\section{A. Abbreviations to Train Comprehension of History}

1) At present, the Chinese historical story is an infrequent mold of game development, which can also slim down the threshold of learning history.

2) Children will not only contact the synopsis of the story before the game but also deepen their understanding in the process of the game if taking historical stories as the background of the game.

\section{B. To Train Strategic Ability}

1) Have a clear thread of the game's tasks and know how to win the game by manipulation.

2) The player is trained to achieve the objective by choosing the applicable game object. On that point are no unique criteria for these choices, nevertheless, different choices contribute different scores.

\section{To Train Motor Coordination Ability}

1) Complete the game by manipulating gestures precisely.

2) Children are required to cooperate with their hands and brain to avoid obstacles and receive the arrow.

Training target of our game is shown in Figure 2.

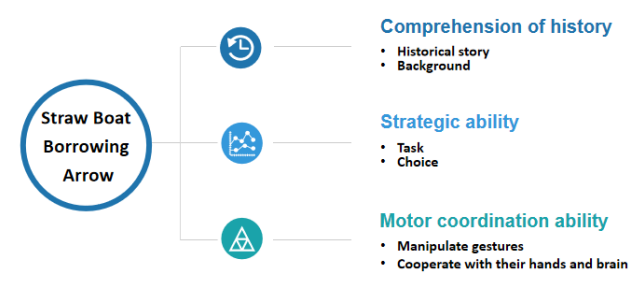

Fig. 2. Training target of Borrowing Arrows with Straw Boats.

Virtual environments as a new medium provide more interesting experiences for games. The objects fired include arrows and arrows with fire, and the game is lost if the arrows with fire are received three times before the required number is reached. Contrariwise, the game wins. Arrows are collected by using gestures to control the movement of the ship, choosing the climate (e.g. foggy, sunny, rainy), the battle site (the Yangtze River and ground), and the equipment (e.g. scarecrow, weapons, soldiers, food, etc.). In the virtual environment, the player uses Leap Motion to track the player's hand-gesture changes.

Leap Motion uses the principle of stereo vision to periodically capture and transmit information about hand movements within a certain range to a computer. For example, if two hands are detected moving in the same direction, they are considered to be translating; if two hands are close together or apart, they are considered to be shrinking or enlarging. Leap Motion as an intermediary for the game, transmitting hand movements to the computer display. The visual nerve of the brain receives and processes the display image while transmitting the judgment of the image and the next operation to the nerve of the hand. The process of information delivery is designed to drive the evolution of the storyline and help players successfully complete their tasks. Leap Motion brings the player and the computer together, which requires the player to match visual and physical movements with each other to play the game and improve children's coordination ability. The system pipeline is shown in Figure 3.

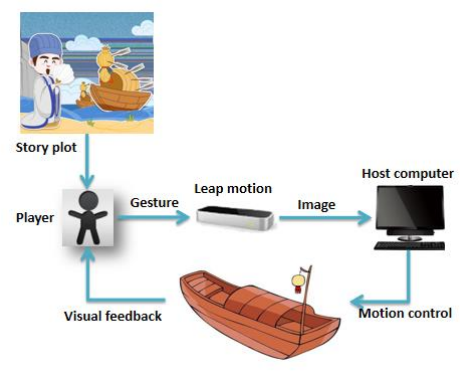

Fig. 3. System pipeline. 
The complete virtual reality system consists of the HTC Vive and Leap Motion as the perception device and tracking device, and participants feel as if they are directing the battle in the field. Participants can choose the climate, battle site and equipment according to their understanding.

Based on Leap Motion and HTC Vive controller, the child's relevant abilities were examined in three aspects: motor performance, sensory performance, and cognitive performance [32]. Children in the game need to manipulate the direction of movement and speed of the boat through hand gestures, which is mainly for the child's motor performance. Visually, if the boat is moved to an area where the arrows fall more densely, the bow will have a higher hit rate, while also risking receiving arrows with fire. When the boat speed matches the visually judged wind speed and direction, it is more favorable for the collection of arrows. This mainly is to train children's sensory performance ability. Identifying arrows and arrows with fire is the key to improving cognitive skills. The player controls the ship to keep moving to receive arrows until the game succeeds or fails. Arrows or arrows with fire lead to different game results. If the number of arrows with fire collected reaches three times, the game is lost, and if the collection of 500 arrows is successfully completed, the game is won.

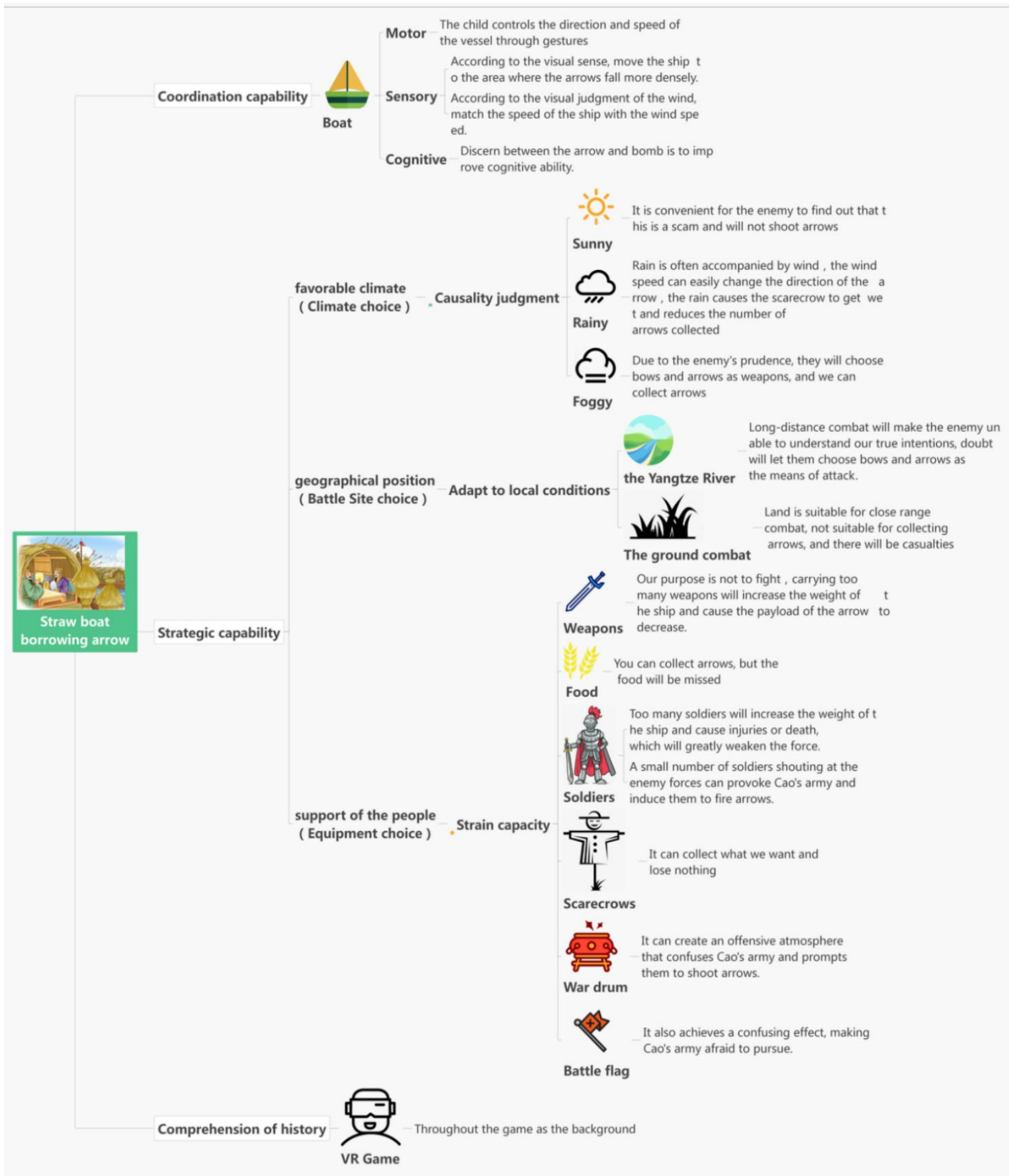

Fig. 4. Capacity Improvement Framework. 
The investigation of strategic capability is from the following three perspectives: favorable climate, geographical position, and support of the people. The ability to analyze the cause and effect of things can be acquired by choosing different game objects. This leaves in their minds the notion that every "outcome" is influenced by the "choices" they have made. This section is designed to develop children's tactical and strategic skills.

A favorable climate refers to the choice of weather. Different weather options have different effects on the game. On a sunny day it is easy to see that this is a hoax, so the enemy will not fire arrows. If the player chooses a rainy day, the rain will get the scarecrow wet. Moreover, there will be wind in rainy days, and the wind speed can easily change the direction of the arrow in rainy days. These phenomena are the reasons that can affect the number of arrows collected. The enemy chose bows and arrows by virtue of prudent when the foggy weather is selected. The time, place, and characteristics of historical stories are the foundation for children to choose the climate.

The choice of battle site trains children's understanding of geography. Players need to pick out a battlefield that suits the current needs based on terrain features and game objectives, thus allowing players to understand the importance of geographic location. Close combat not only hinders the collection of arrows, but also causes casualties, as swords are often used as weapons in ground combat. When players choose to fight on the Yangtze River, they can provoke their enemies by bluffing. The enemy could not figure out our true intentions and chose the arrows as a means of attacking from a distance, so we could take advantage of this opportunity to silently collect more arrows.

The choice of equipment on board is based on whether the equipment helps achieve the game goal. We provide equipment that can be put on board, including Weapons, Food, Soldiers, Scarecrows, War Drums and Battle Flags. Carrying enough weapons will not help the player achieve the goal of collecting arrows and will increase the weight of the ship. Piling grain together in huge targets facilitates the hit rate of arrows. However, this process can lead to food waste. When the soldiers shout at the enemy, they can provoke Cao's army and induce them to shoot arrows. Scarecrows can help us get what we want with no loss. The use of drums can create an offensive atmosphere, confusing Cao's army and prompting them to shoot arrows. The reason why Cao's army did not dare to fight at close range was because the battle flag and war drums had a similar effect and could mislead the enemy.

The training of traditional Chinese culture is carried out throughout the game and is not reflected in any one part of the game. The story as the background of the game will be provided to the player before the game starts. During the game, players can have a deep understanding and knowledge of the details in the story. Capacity improvement framework shown in Figure 4.

\section{IMPLEMENTATION}

Combining the four interrelated activities of Klabbers [33] and the four-dimensional framework proposed by de Freitas, S
[34], we propose a new educational framework which includes interaction, presentation, and schemas. The details of the game were implemented according to the design focus of the game and the new educational framework. The new education structure consisted of three aspects: interaction, presentation, schema.

First, teacher-student interaction is one of the important components of the framework. The teacher is not only an educator, but also an organizer who plays a leading role in the teaching and learning process. Whether teacher-student interaction takes place or not depends not only on the identity and role of the teacher, but also on the degree of cooperation of the students themselves, and whether the teacher's lecture style is appealing to the students. Young people between the ages of 6 and 12 have a strong sense of curiosity, and capitalizing on this characteristic of theirs enhances student engagement by capturing their attention through engaging methods rather than rigid text. The self-operated interactive game (Figure 5) serves as a medium for teacher-student communication, based on depth perception technology (Figure 6), which not only helps teachers to demonstrate intuitive, engaging, and actionable knowledge points, but also presents the Battle of Red Cliff to students in a novel format.
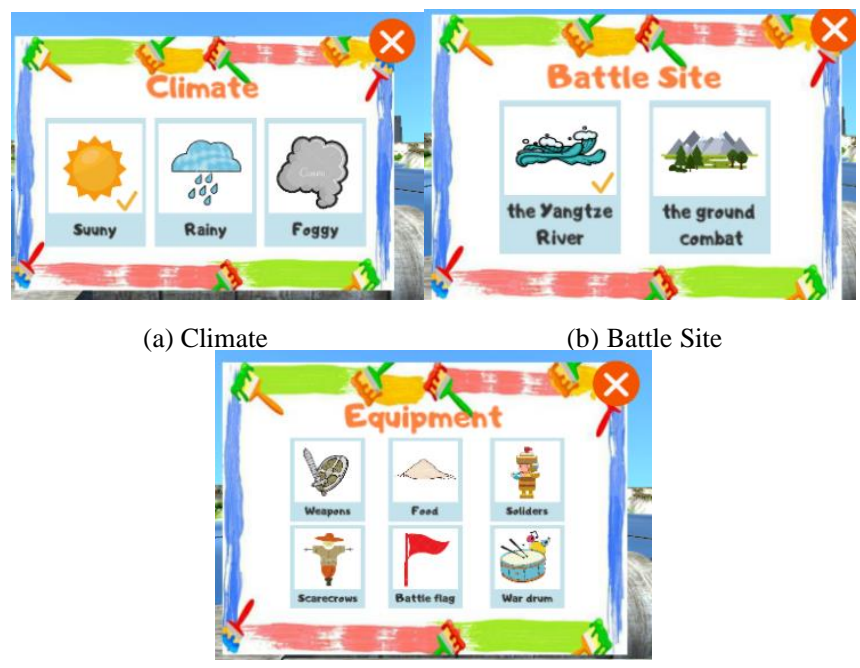

(c) Equipment

Fig. 5. Game selection interface.

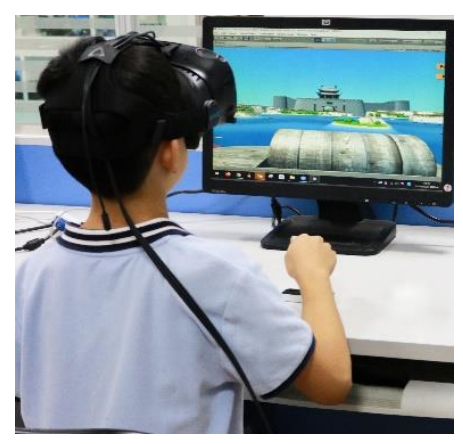

Fig. 6. A child who used HTC Vive to play games.

The second dimension focuses on the form of educational presentation. Single-media forms of instruction such as text or pictures are about ten percent effective; highly immersive 
media such as VR instruction can be up to seventy percent effective. The game combines various elements through Unity to build a realistic and interesting game interface. The game interface can present a scene in which a large number of arrows and a few arrows with fire are shot at the same time, and the ship can move back and forth as a collection object. In addition to the screen design of the game, we also provide a choice of climate, battle site and equipment, as shown in Figure 7(a) for a rainy day, which contrasts with the sunny day in Figure 7(b). Learners can put themselves into the story scenario and experience the whole process of historical events in the game as the main character. The plot and graphics presented in the game involve a variety of senses rather than the imagination, and the immersion that comes with this process naturally improves their learning efficiency.

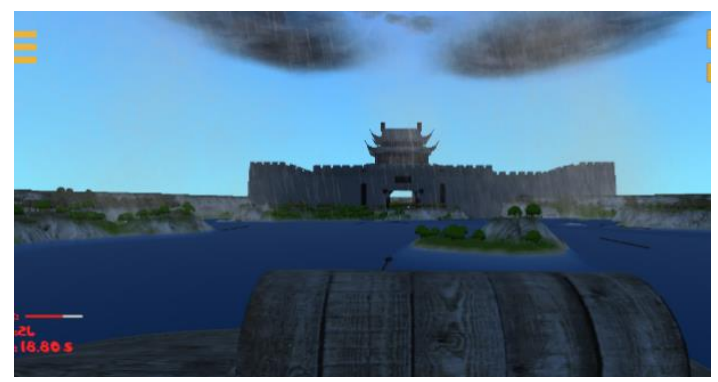

(a) Rainy

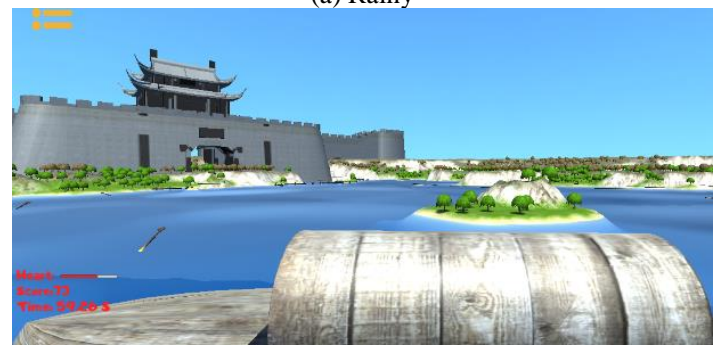

(b)Sunny

Fig. 7. Different weather in game.

Schemas integrates the topics designed and the teaching tools used. Traditional Chinese culture is unrepeatable and untouchable, so we can only observe history through written descriptions. And virtual reality technology has the advantages of immersion, conceptualization, and interactivity. Based on the characteristics of both, we will create a new teaching environment for learners that combines virtual reality technology with traditional Chinese culture education. Learners understand the essence of the game's expression because of the immersive experience that comes with realistic scenes.

These three dimensions form the framework of this game design, and each latitude in this framework is inextricably linked. They are interconnected and interact with each other, providing more possibilities for learning from multiple perspectives. The game-based learning designed through this framework considers both student and teacher perspectives, increasing interaction between teachers and students and improving learning efficiency.

The whole game is divided into two steps. The first step is to set the game scenario and select the options that will help to complete the game tasks. Our main research subjects are three modules, each of which offers some options, namely Battle site options: the Yangtze River and the ground combat. Climate options: sunny, rainy, and foggy. Equipment options: weapons, food, soldiers, scarecrows, war drums, and battle flags. The second step is to control the movement of the boat with hand gestures. After the game object selection is finished, you can start collecting arrows. After that, we have to move the boat according to the wind direction, the direction of enemy's attack, the concentration of fog, etc. to make sure the boat can finish collecting arrows.

\section{EvALUATION}

We recruited seventy students aged 6-12 years to evaluate the effectiveness of our educational games in improving historical comprehension ability, strategic ability, and motor coordination ability. Different competency training was tested with different indicators. We divided the 70 children equally into two groups for comparison, i.e., those trained with and without virtual reality.

The Wechsler Intelligence Scale for Children (WISC) [35] and Movement Assessment Battery for Children-Second Edition (MABC-2) [36] were used to evaluate the educational game as our choice. WISC, developed by David Wechsler, is an independently managed intelligence test tool widely used by children aged 6 to 16. It provides four subscales of Perceptual Reasoning (PR) to assess strategy and problem solving skills. The Reasoning scale consists of four subscales: Block Design, Picture Concepts, Matrix Reasoning, and Picture Completion. This article uses the Chinese version of WISC-IV, which is more in line with the language habits of Chinese children. The MABC-2, published by Pearson, has been called the "Gold Standard Test" for assessing children's motor coordination. Standardized tests are used to measure motor coordination in children aged 4-16 years worldwide. The use of measurement instruments in the MABC-2 is more in line with the developmental status of children.

As a scripting language, Python [37], [38] has many extension libraries for scientific computing that provide Python with fast array processing, numerical operations, and plotting capabilities. In addition, some of the reasons we chose the language were its simplicity, readability, and scalability. After analyzing the data using Python, we chose Z-test [39]-[41] to evaluate the data. A Z-test is any statistical test for which the distribution of the test statistic under the null hypothesis can be approximated by a normal distribution. In general, many statistical tests can be conveniently performed as approximate Z-tests if the sample size is large $(n>30)$ or the overall variance is known.

According to the specific scores of the WISC-IV and MABC-2, we have plotted the two sets of data separately, where $\mathrm{A}$ and $\mathrm{B}$ are the results after training with and without VR. From the results of the drawing (Figure 8), the distribution of data approximately follows the typical pattern for all normal distributions. Also, since the sample size is greater than 30, an approximate Z-test can be easily performed. Calculate the average and variance of the two groups A and B (Table I) through the mean() and $\operatorname{std}()$ functions, we extrapolated that the test results using VR are better and perform hypothesis 
testing. As a rule, we put forward two opposing assumptions: the alternative hypothesis and the null hypothesis. When the null hypothesis is true, the alternative hypothesis is false. In Ztest, we usually choose 0.05 as a confidence interval for rejecting the null hypothesis and accepting the alternative hypothesis. According to Table II can be obtained that the results of Z-test with Python. The p-value calculated by the ztest() function of the two groups are far less than 0.05 , so we accept the alternative assumptions, i.e., children's abilities have higher improved after VR training.
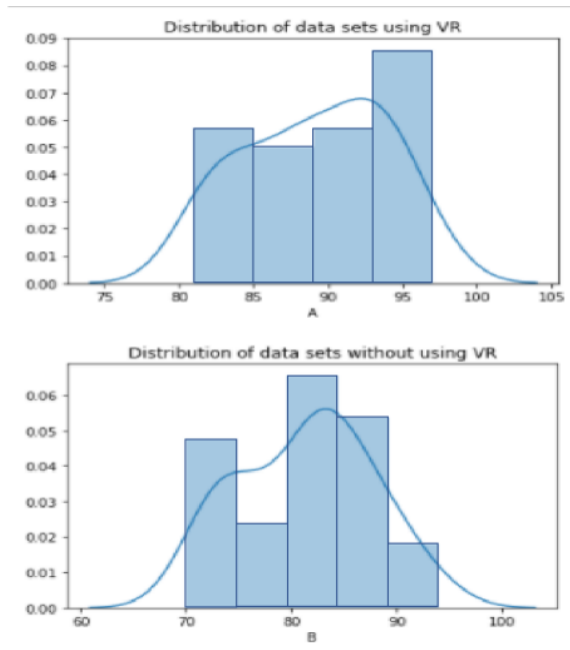

(a) WISC-IV
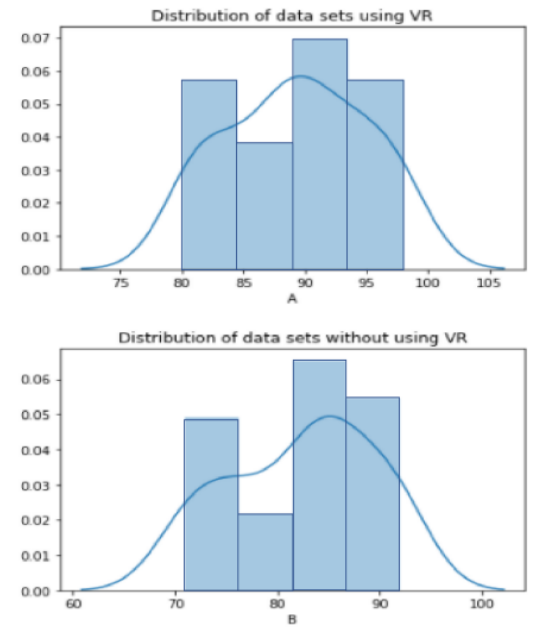

(b) MABC-2

Fig. 8. The distribution of data.

TABLE I. The Average AND VARIANCE of THE Two Groups A AND B

\begin{tabular}{|c|c|c|c|}
\hline & & Mean & Std \\
\hline \multirow{2}{*}{ WISC-IV } & $\begin{array}{c}\text { A (training with } \\
\text { VR) }\end{array}$ & 89.171429 & 4.804934 \\
\cline { 2 - 4 } & $\begin{array}{c}\text { B (training } \\
\text { without VR) }\end{array}$ & 81.342857 & 6.291651 \\
\hline \multirow{2}{*}{ MABC-2 } & $\begin{array}{c}\text { A (training with } \\
\text { VR) }\end{array}$ & 89.314286 & 5.608667 \\
\cline { 2 - 4 } & $\begin{array}{c}\text { B (training } \\
\text { without VR) }\end{array}$ & 82.4 & 7.005040 \\
\hline
\end{tabular}

TABLE II. THE TEST RESUlts OF Z-TEST

\begin{tabular}{|c|c|c|c|}
\hline $\begin{array}{c}\text { Congnitive } \\
\text { skills }\end{array}$ & $\begin{array}{c}\text { Accept } \\
\text { alternative } \\
\text { Hypothesis }\end{array}$ & $\begin{array}{c}\text { p-value in } \\
\text { z-test }\end{array}$ & $\begin{array}{c}\text { Accept/Reject } \\
\text { alternative } \\
\text { Hypothesis }\end{array}$ \\
\hline $\begin{array}{c}\text { Strategic } \\
\text { (WISC-IV) }\end{array}$ & $\begin{array}{c}\text { Strategic score } \\
\text { increased after } \\
\text { VR training }\end{array}$ & $\begin{array}{c}4.9066 \mathrm{e}-09 \\
<0.05\end{array}$ & Accept \\
\hline $\begin{array}{c}\text { Motor } \\
\text { coordination } \\
\text { (MABC-2) }\end{array}$ & $\begin{array}{c}\text { Motor } \\
\text { coordination } \\
\text { score increased } \\
\text { after VR } \\
\text { training }\end{array}$ & $\begin{array}{c}5.1555 \mathrm{e}-06 \\
<0.05\end{array}$ & Accept \\
\hline
\end{tabular}

In addition to testing strategic ability and motor coordination ability, we designed a set of questions to assess children's understanding and mastery of historical stories, their acceptance of virtual reality, and the playability of the game. The impact of educational games on children's learning was evaluated from different perspectives. The findings in Figure 9 show that some children may be new to virtual reality games and are not very proficient in virtual reality games. However, each child's acceptance of virtual reality is high, which indicates that gamification of education is very attractive to children. Most students were able to master history knowledge, but only a small percentage of students were able to achieve the best results. Combining all the data from the questionnaire, the results of the questionnaire can be summarized into four areas, which are:

- The combination of VR and education is more attractive to students;

- Historical knowledge is the basic aim of training;

- Students have to practice more with new equipment;

- How to make students pay more attention to the mastery of knowledge is needed for further study.

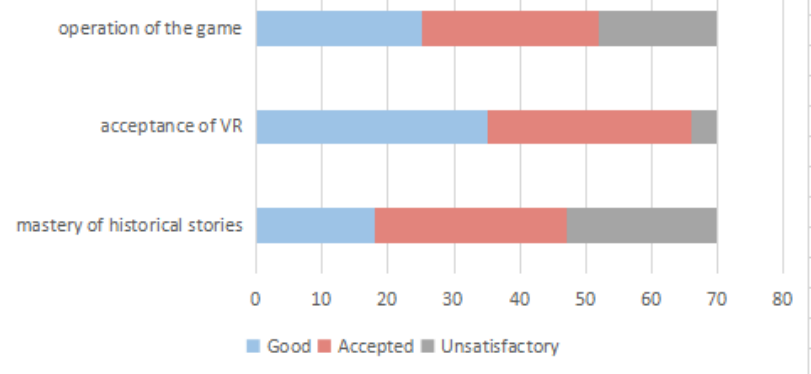

Fig. 9. Distribution of survey results.

\section{DISCUSSION}

Educational gamification is a common teaching method nowadays. This kind of teaching method is no longer limited to books and boring teaching models in the classroom, but brings new experiences to children in a lively and fun way. In this era, our country needs children with all-round abilities. This is a serious game that uses Leap Motion and HTC Vive to produce powerful interactivity. After a series of tests, we found from the experiments that the design of this game is more attractive to children. Using interactive devices to control the movement of the boat can improve children's motor coordination ability. At the same time, borrowing arrows from the grass boat as the 
background of the game can also facilitate the grasp of historical knowledge. The new devices are applied to more game design, which can improve children's learning enthusiasm. Through the combination of virtual reality technology and traditional culture, new technology is used to transfer old knowledge and help children look back at history. This paper just selects a short story that happened during the Three Kingdoms period as an example. We hope to introduce more traditional Chinese culture in future research and contribute to the heritage of Chinese culture.

\section{ACKNOWLEDGMENT}

The research leading to these results has received funding from the Scientific and Technological Project in Henan Province, China (No. 202102210397). The authors acknowledge partial support from National Natural Science Foundation of China (NSFC, 61802352).

\section{REFERENCES}

[1] Z. Huang, A. Javaid, V. K. Devabhaktuni, Y. Li and X. Yang, "Development of Cognitive Training Program With EEG Headset," in IEEE Access, vol. 7, 2019, pp. 126191-126200.

[2] P. J. Lamont, "Cognitive decline in a young adult with pre-existent developmental delay-What the adult neurologist needs to know," Practical Neurology, vol. 4, no. 2, 2004, pp. 70-87.

[3] K. Ball, J. D. Edwards, L. A. Ross, and G. McGwin, "Cognitive training decreases motor vehicle collision involvement of older drivers," J. Amer. Geriatrics Soc., vol. 58, no. 11, 2010, pp. 2107-2113.

[4] D. L. King and P. H. Delfabbro, "The cognitive psychology of Internet gaming disorder," Clin. Psychol. Rev., vol. 34, no. 4, 2014, pp. 298308.

[5] N. Schneider and C. Yvon, "A review of multidomain interventions to support healthy cognitive ageing," J. Nutrition, Health Aging, vol. 17, no. 3, 2013, pp. 252-257.

[6] R. S. Leder, N. Murillo, C. P. Ibarra, et al. "Computer game motivating rehabilitation with objective measures of improvement in motor function", 2001 Conference Proceedings of the 23rd Annual International Conference of the IEEE Engineering in Medicine and Biology Society. IEEE, 2001, 2: 1388-1390.

[7] T. W. Malone. "What makes things fun to learn? A study of intrinsically motivating computer games", 1981.

[8] T. Partovi, M. R. Razavi. The effect of game-based learning on academic achievement motivation of elementary school students [J]. Learning and Motivation, 2019, 68: 101592.

[9] A. C.Siang, R. K. Rao. "Theories of learning: a computer game perspective", Fifth International Symposium on Multimedia Software Engineering, 2003. Proceedings. IEEE, 2003, pp. 239-245.

[10] https://images.app.goo.gl/f6xYGMbRXwJpEp9Z9

[11] J. Fiber. "The Future of VR \& (Non-Gaming) Entertainment", TWICE, 2016, 31(16)

[12] I. Gace, L. Jaksic, I. Murati, I. Topolovac, M. Zilak and Z. Car, "Virtual Reality Serious Game Prototype for Presenting Military Units," 2019 15th International Conference on Telecommunications (ConTEL), Graz, Austria, 2019, pp. 1-6.

[13] D. L. Zhang. "Improvement of Medical Clinical Teaching by VR Medical Surgical Helmet", Institute of Management Science and Industrial Engineering. Proceedings of 2019 3rd International Conference on Economics, Management Engineering and Education Technology (ICEMEET 2019). Institute of Management Science and Industrial Engineering: (Computer Science and Electronic Technology International Society), 2019, pp. 1972-1974.

[14] X. G. Hei. "An Affordable Motorized Generation System of Object VR Movie", Intelligent Information Technology Application Association. Proceedings of the 2011 International Conference on Informatics,
Cybernetics, and Computer Engineering (ICCE 2011 V3). Intelligent Information Technology Application Association, 2011, pp. 81-89.

[15] S. C. Chang, T. C. Hsu, M. S. Y. Jong. "Integration of the peer assessment approach with a virtual reality design system for learning earth science", Computers \& Education, 2020, 146: 103758.

[16] H. Tüzün, M. Yılmaz-Soylu , T. Karakuș, et al.. "The effects of computer games on primary school students' achievement and motivation in geography learning", Computers \& Education, 2009, 52(1): 68-77.

[17] C. Evangelista, M. Carrozzino, V. Neri and M. Bergamasco, "Interactive Storytelling for Children Education," 2009 Conference in Games and Virtual Worlds for Serious Applications, Coventry, 2009, pp. 198-201.

[18] J. Froschauer, M. Arends, D. Goldfarb and D. Merkl, "Towards an Online Multiplayer Serious Game Providing a Joyful Experience in Learning Art History," 2011 Third International Conference on Games and Virtual Worlds for Serious Applications, Athens, 2011, pp. 160-163.

[19] G. Dzhambazov and K. Goranchev, "Sing Master: An Intelligent Mobile Game for Teaching Singing," 2016 8th International Conference on Games and Virtual Worlds for Serious Applications (VS-GAMES), Barcelona, 2016, pp. 1-2.

[20] C. Voutounos and A. Lanitis, "On the Presentation of Byzantine Art in Virtual Environments," 2011 Third International Conference on Games and Virtual Worlds for Serious Applications, Athens, 2011, pp. 176-177.

[21] E. Pietroni, A. Pagano, and C. Rufa, "The etruscanning project: Gesturebased interaction and user experience in the virtual reconstruction of the regolini-galassi tomb," in 2013 Digital Heritage International Congress (DigitalHeritage), vol. 2, 2013, pp. 653-660.

[22] C. Zhou, H. Li and Y. Bian, "Identifying the Optimal 3D Display Technology for Hands-On Virtual Experiential Learning: A Comparison Study," in IEEE Access, vol. 8, pp. 73791-73803, 2020.

[23] S. Rizvic et al., "Guidelines for interactive digital storytelling presentations of cultural heritage," 2017 9th International Conference on Virtual Worlds and Games for Serious Applications (VS-Games), Athens, 2017, pp. 253-259.

[24] J. M. Fernandes and S. M. Sousa, "PlayScrum-A Card Game to Learn the Scrum Agile Method," 2010 Second International Conference on Games and Virtual Worlds for Serious Applications, Braga, 2010, pp. 52-59.

[25] O. Philbin-Briscoe et al., "A serious game for understanding ancient seafaring in the Mediterranean sea," 2017 9th International Conference on Virtual Worlds and Games for Serious Applications (VS-Games), Athens, 2017, pp. 1-5.

[26] D. Christopoulos, P. Mavridis, A. Andreadis and J. N. Karigiannis, "Using Virtual Environments to Tell the Story: "The Battle of Thermopylae"," 2011 Third International Conference on Games and Virtual Worlds for Serious Applications, Athens, 2011, pp. 84-91.

[27] K. Kiili. "Digital game-based learning: Towards an experiential gaming model", The Internet and higher education, 2005, 8(1): 13-24.

[28] M. Zyda. "From visual simulation to virtual reality to games",Computer, 2005, 38(9): 25-32.

[29] N. Peirce, O. Conlan and V. Wade, "Adaptive Educational Games: Providing Non-invasive Personalised Learning Experiences," 2008 Second IEEE International Conference on Digital Game and Intelligent Toy Enhanced Learning, Banff, BC, 2008, pp. 28-35.

[30] S. Rizvic and I. Prazina, "Taslihan Virtual Reconstruction - Interactive Digital Story or a Serious Game," 2015 7th International Conference on Games and Virtual Worlds for Serious Applications (VS-Games), Skovde, 2015, pp. 1-2.

[31] K. Kiili, S. de Freitas, S. Arnab, T. Lainema, "The Design Principles for Flow Experience in Educational Games,". Procedia Computer Science, 2012.

[32] A. Asadipour, K. Debattista and A. Chalmers, "A Game-Based Training Approach to Enhance Human Hand Motor Learning and Control Abilities," 2015 7th International Conference on Games and Virtual Worlds for Serious Applications (VS-Games), Skovde, 2015, pp. 1-6.

[33] Pereira, L. Luís, G. Licínio . Roque. "Design Guidelines for Learning Games: the Living Forest Game Design Case." DiGRA Conference, 2009. 
[34] D. Freitas, S. Oliver, M. (2006). "How can exploratory learning with games and simulations within the curriculum be most effectively evaluated?" Computers and Education, Special Issue. 2006, pp. 249-264.

[35] X. Bu, R. Qiao and D. Han, "Thinking Ability Visualization Model Based on Wechsler Intelligence Scale for Children," 2014 Sixth International Conference on Intelligent Human-Machine Systems and Cybernetics, Hangzhou, 2014, pp. 237-240.

[36] C. Outer, D. M. Kristine, C. Samaey, et al Movement skill assessment of typically developing preschool children: a review of seven movement skill assessment tools J Sports Sci Med, 2008, 8(6): 154-168.

[37] L. Yu, "Empirical Study of Python Call Graph," 2019 34th IEEE/ACM International Conference on Automated Software Engineering (ASE), San Diego, CA, USA, 2019, pp. 1274-1276.

[38] P. Dinesh Divsekar, R. Porob and P. S. Kuwelkar, "Automated Regression Testing and Data Analytics using Python," 2019 4th
International Conference on Recent Trends on Electronics, Information, Communication \& Technology (RTEICT), Bangalore, India, 2019, pp. 95-99.

[39] E. B. Fatima and E. M. Abdelmajid, "Study of efficiency k-means clustering using Z-test proprieties," 2017 Intelligent Systems and Computer Vision (ISCV), Fez, 2017, pp. 1-5.

[40] C. Li, "Z-test of computer-and-internet-aided multimodal English listening and speaking," 2013 IEEE Third International Conference on Information Science and Technology (ICIST), Yangzhou, 2013, pp. 98101.

[41] R. K. Shial, B. Sankar Gouda, S. R. Pattanaik and N. Sethi, "A Centralized Faulty Node Detection Algorithm Based on Statistical Analysis in WSN," 2020 International Conference on Computer Science, Engineering and Applications (ICCSEA), Gunupur, India, 2020, pp. 1-6. 